\title{
It's not what you hear but how often you hear it: On the neglected role of phonological variant frequency in auditory word recognition
}

\author{
CYNTHIA M. CONNINE \\ State University of New York, Binghamton, New York
}

\begin{abstract}
Recognition of a frequently heard spoken word variant in American English (flapping) was investigated in a phoneme identification experiment. Listeners identified the initial segment $(b$ or $p)$ of word-nonword continua (e.g., pretty-bretty) that was embedded in either a flap or a [t] variant carrier word (e.g., preDy-breDy or preTTy-breTTy). The results showed more identification responses forming a real word when the to-be-identified speech sound occurred in the more frequently experienced flap carrier. These results support the claim that lexical representation of spoken words includes the flap variant. Listeners do not recode the flap variant into an underlying $/ \mathrm{t} / \mathrm{version}$ but recognize the flap, in its surface form, via a preexisting representation in lexical memory.
\end{abstract}

Successful spoken language processing involves coordinated comprehension among speakers. A primary interface between the speech signal and comprehension is a representation of words in a vocabulary based on form (phonetic features). In order to recover the semantic content of an utterance or a discourse, a listener must successfully map the speech input to a form-based representation. The mapping between the input and a lexical representation is crucial because it provides a gateway to a word's meaning and structural properties. The success of the mapping process depends on recovery of relevant information from the signal, as well as the degree to which the representation of the input is compatible with a stored lexical representation.

Theories of spoken word recognition must account for the fact that spoken language exhibits considerable variability in the physical speech signal. Sources of variability in speech include individual speaker properties, as well as phonetic category and lexical characteristics. For speaker characteristics, a large body of research suggests that the indexical properties of spoken words are encoded and retained in memory (Goldinger, 1998; Mullennix, 1997). For phonetic category, Miller (2001) has shown that listeners encode the variability found within a speech category, with some category members being perceived as better exemplars than other members are.

Variability of spoken words as a function of lexical characteristics is also found. One class of phonological processes results in systematic variation in the speech

The research was supported by NIH Grant R01DC02134. I thank Albrecht Inhoff and Larissa Ranbom for helpful comments on the manuscript and David Patterson for his assistance in conducting the experiment. Address correspondence to C. M. Connine, State University of New York, Department of Psychology, Binghamton, NY 13901 (e-mail: connine@binghamton.edu). signal that is licensed for particular lexical environments. A systematic variant in American English, flapping, can occur when a voiceless alveolar stop follows a stressed vowel (e.g., the word pretty may be realized as [prirı]). The present research focuses on the mechanism for recognizing phonological variants - specifically, American English flaps - and investigates the claim that frequently heard production variants are explicitly represented in lexical memory. Note that the notion of variant frequency differs from conventional frequency metrics in that the dimension of interest is the production frequency of a particular phonological variant relative to an alternative.

One hypothesis for how listeners process phonological variants during spoken word recognition assumes that special inferential mechanisms recover underlying representations from a distorted speech. This view has been proposed for contextually conditioned variants, such as place assimilation (e.g., lean $\rightarrow$ leam when followed by bacon; Gaskell \& Marslen-Wilson,1996), and for schwa vowel deletion (e.g., suppose $\rightarrow$ s'pose; LoCasto \& Connine, 2002). On this view, listeners recode the speech signal on the basis of language knowledge into a representation that matches a representation stored in the lexicon.

Inference-based models seem plausible for phonological variants that occur relatively infrequently. Although no frequency statistics are available for place assimilation, phonological variation requiring a specific combination of word endings and beginnings (e.g., the $/ \mathrm{n} /$ in lean followed by the /b/ in bacon) is unlikely to be frequent. For schwa vowel deleted variants, a recent corpus analysis of conversational speech (Patterson, LoCasto, \& Connine, 2003) showed that two-syllable words were produced rarely $(11 \%)$ as schwa vowel deletion variants. The infrequent occurrence of schwa deleted words would render an inference-based mechanism appealing. However, inference processes for low-frequency variants may be time and re- 
source consuming, as well as error prone. Consistent with this claim, LoCasto and Connine (2002) found that lexical decisions to schwa vowel deleted variants were responded to more slowly and had higher error rates than their vowel-bearing counterparts. Similarly, deleted forms had lower goodness ratings and were more often categorized as nonwords in goodness of production ratings.

An inference-based mechanism loses some appeal when one considers more frequent variants. For American flaps, the overwhelming intuition for native speakers is that the flap predominates in casual conversation. Consistent with intuitions about flap productions is their linguistic characterization as the regular pronunciation (Akmajian, Demers, \& Harnish, 1984). These observations have been examined empirically in a corpus analysis (Patterson \& Connine, 2001) in which productions of potentially flapped words were extracted from a conversational database (Godfrey, McDaniel, \& Holliman, 1992). Of 2,172 tokens from 155 speakers in the database, 2,088 $(96 \%)$ consisted of unambiguous flapped productions. The remaining 4\% consisted of articulated [t] and glottal stop productions. The predominance of the flapped production held for all the words and for two different dialect regions.

The sheer frequency of flapped productions would suggest that processing efficiency would be maximized via an explicit representation of the flap in lexical memory. Rather than performing a potentially time- and resourceconsuming recoding of flaps into the underlying $/ \mathrm{t} /$ form, recognition of flapped variants would be accomplished by an explicit representation in the lexicon. This view is consistent with the wide body of evidence showing that listeners encode and retain a great deal of surface detail from individual speakers (Mullennix, 1997), as well as from individual speech sounds (Miller, 2001). The notion that lexical representations encode detailed surface representations is not without detractors. Some have argued that lexical representations are highly abstract (Marslen-Wilson, Nix, \& Gaskell, 1995). The abstract representation view was developed to account for the processing of contextually conditioned phonological variation (place assimilation across word boundaries) and has not been explicitly tested on word-internal phonological variation. On this view, processing of flap and [ $t$ ] variants would be processed comparably, since the lexical representations are designed to accommodate either form.

The hypothesis tested here is whether representation of lexical form includes a representation of the highly frequent American English flap. A phoneme identification task was used in which the target segment was either an unambiguous or an ambiguous token of a speech sound (Ganong, 1980). In the variant of the task used here, listeners identified the initial segment of word-nonword speech continua, which was embedded in either a flap or a [t]-bearing carrier word (e.g., preDy-breDy or preTTybreTTy). Both p-lexical-bias (e.g., pretty-bretty) and blexical-bias words (e.g., beetle-peetle) were used. If the more frequent, flapped form is represented in lexical memory, the flapped carrier word should show more identification responses that form a real word than the [t] carrier word would. More specifically, b-bias stimuli should show more $b$ (word-forming) responses for the flap than for the $[\mathrm{t}]$ carrier. The opposite pattern should be found for the p-bias words with fewer $b$ (more $p$ or word-forming) responses for the more frequent flap. The abstract representation view assumes that the two carrier stimuli are treated equivalently and predicts no difference for the flap and the [t] carriers.

\section{METHOD}

\section{Participants}

Two groups of 18 undergraduates at Binghamton University participated to fulfill a class requirement. All the participants were native speakers of English and reported normal hearing.

\section{Materials and Design}

Six words with medial $[t]$ segments that could appear as flapped segments were selected. Three words began with a voiced segment, and three words began with a voiceless segment. For each word, a nonword could be created by altering the voicing value of the initial segment (e.g., beetle-peetle). A seventh pair was selected as a practice stimulus (button-putton). Each word/nonword pair was recorded in a soundproof booth by a female native American English speaker, one with a medial $[\mathrm{t}]$ pronunciation and the second with a [r] (flap) pronunciation. Stimuli were recorded in digital form directly onto the hard disk of a computer $(44 \mathrm{kHz}, 16$-bit resolution). Productions of the flap and the [t] variants were selected that had similar durations of aperiodic energy for the initial voiceless segment (e.g., the voice onset times for the production of beetle and beedle were similar). Selections of the flap and the [t] variant productions were similarly matched.

Measurements of the preceding vowel showed longer vowels for the [t] than for the flap production in four stimuli (better, bottle, barty, and battern). Given that differences in vowel length could influence the results in unpredictable ways, a second set of stimuli were constructed in which vowel length was equated across the two productions. In the vowel-equated set, the vowel from the flapped production was decreased in length to the average duration of the original flap and $[t]$ vowel lengths by deleting whole pitch periods from the center of the vowel. The resulting vowel segment was spliced into both the flapped and the [t] productions. A similar procedure was followed for the two remaining stimulus pairs (beetle and bretty, differing by 2 and $3 \mathrm{msec}$, respectively) to maintain consistency across the stimulus set. In this way, the vowel-equated stimuli had identical first-syllable vowels (and surrounding consonants) across the flap and $[t]$ continua. Two phonetically trained listeners confirmed that the flap and the $[t]$ versions were unambiguous with respect to the voicing value of the medial segment.

A voicing continuum was created for the flap and [t] stimulus pairs. Successive portions of periodic energy were excised from the voiced stimulus, starting from the end of the closure of the voiced segment. These portions were replaced with equal-length acoustic portions of aperiodic energy taken from the voiceless stimulus, starting from the end of the closure. The identical portions were spliced into the flap and [t] stimuli. All splicing was done at zero crossings in order to avoid abrupt changes in amplitude. Successive portions differed by approximately $5 \mathrm{msec}$. In order to select stimuli from the midrange of the continua for presentation in the experiment, a pilot study was conducted with 5 listeners, using the entire set of stimuli. Four midrange stimuli that the listeners perceived as ambiguous were selected. 
The four midrange ambiguous stimuli, as well as the unambiguous endpoints, were presented (see the Appendix). The original and vowel-equated stimuli were presented to two different groups of participants (18 participants in each group). For both groups of participants, presentation was blocked so that [t] and flap carrier continua for a given word were presented in a single block. A block for a given word contained the six stimuli from each of the two phonological variant continua (flap, $[\mathrm{t}]$ ) repeated six times in random order. A warning tone of $100 \mathrm{msec}$ that was followed by $300 \mathrm{msec}$ of silence preceded each stimulus. Trials were separated by $2 \mathrm{sec}$ of silence. Presentation of word order was randomized across participants. The participants were randomly assigned to either the original or the vowel-equated stimulus groups.

\section{Procedure}

The participants were run individually in a quiet room. All the stimuli were presented binaurally over headphones (Sennheiser HD-250 II). The instructions directed the participants to indicate whether the stimulus they heard began with $\mathrm{a} / \mathrm{b} /$ or a $/ \mathrm{p} /$ sound by circling the corresponding letter on the response sheet. Breaks were provided at the end of each word/nonword pair block. Prior to the experiment, a short practice session was presented. The entire experiment lasted approximately $25 \mathrm{~min}$.

\section{RESULTS AND DISCUSSION}

Figure 1 shows the percentage of voiced $(b)$ responses for each stimulus token (six tokens per continuum) as a function of lexical bias (b-bias vs. p-bias) and carrier type (flap vs. [t]). A clear variant frequency effect was found: Listen- ers were more likely to identify the initial segment as consistent with the lexical bias (forming a real word) when it occurred in the flap carrier, as compared with the [t] carrier. This was found for both lexical bias conditions, with a somewhat larger effect for b-bias words. The variant frequency effect was confined to the intermediate tokens (Tokens 2, 3, 4, and 5), since the endpoints were not ambiguous.

A 2 (lexical bias) $\times 2$ (carrier type) $\times 4$ (intermediate stimulus tokens) $\times 2$ (original/vowel duration equated stimulus group) analysis of variance was conducted across participants $\left(F_{1}\right)$ and items $\left(F_{2}\right)$. Lexical bias was treated as a between-items variable for item analyses, and stimulus group was treated as a between-groups variable for item and participant analyses. Unless otherwise indicated, a significance level of $p<.05$ was used. No main effect of stimulus group was found $(F<1)$, but stimulus group interacted with stimulus token in the participants analysis $\left[F_{1}(3,102)=4.4, M S_{\mathrm{e}}=302 ; F_{2}, p=.23\right]$. The vowelequated stimulus group tended to show a larger percentage of $b$ responses, as compared with the original stimulus group, for positions 2 and 3 ( $9 \%$ and $2 \%$ differences, respectively). This trend was reversed in positions 4 and 5, with the vowel-equated stimulus group showing somewhat fewer $b$ responses, as compared with the original stimulus group ( $4 \%$ and $5 \%$ differences, respectively).

Critically, the lexical bias $\times$ carrier type interaction was significant $\left[F_{1}(1,34)=19.8, M S_{\mathrm{e}}=146, p<.05\right.$;
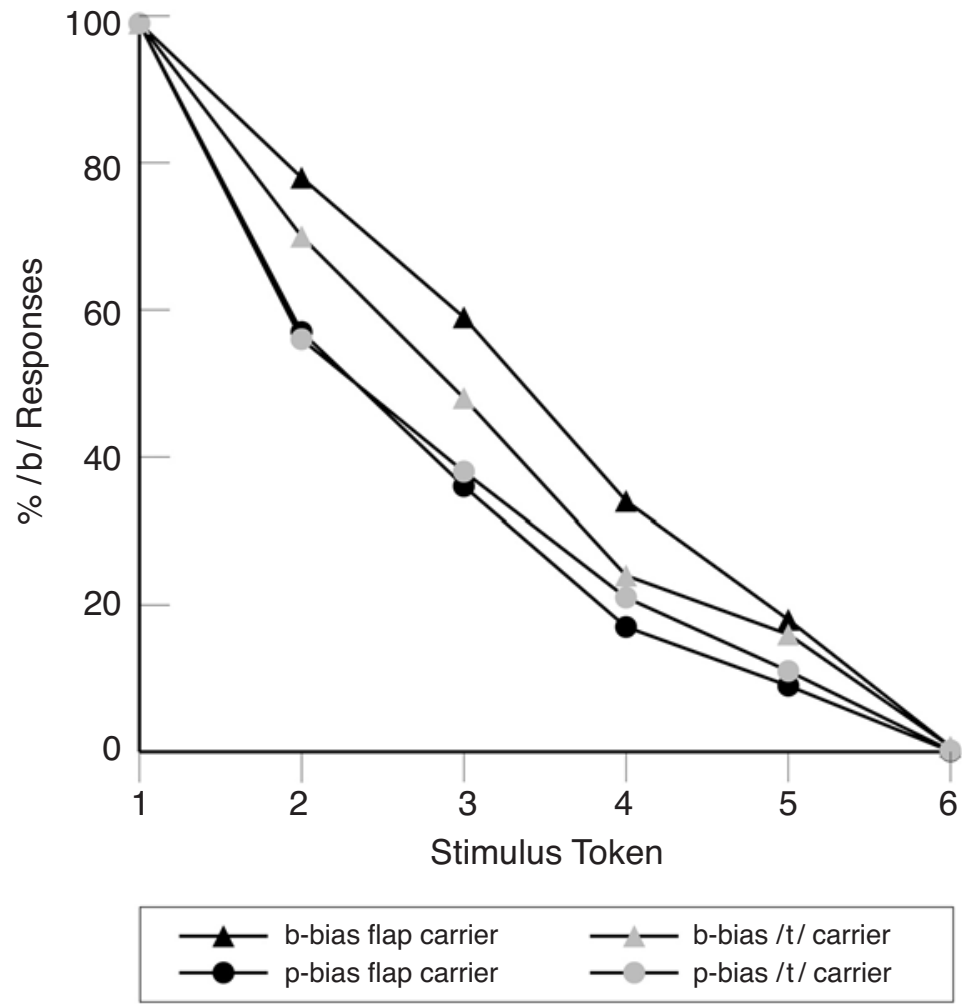

Figure 1. Percentage of voiced /b/ responses as a function of stimulus token (1-6), lexical bias (b-bias or p-bias), and variant carrier (flap or /t/ carrier). 
$\left.F_{2}(1,8)=4.8, M S_{\mathrm{e}}=99, p=.059\right]$. As was predicted, there were more $b$ responses for b-bias words in the flap $(48 \%)$ than in the $[\mathrm{t}]$ carriers $(40 \%)$. The p-bias words also showed the predicted (but somewhat smaller) variant frequency effect: fewer $b$ responses (more $p$ or word responses) were found for the flap carrier (30\% vs. 33\%, flap vs. [t] carrier). A main effect for stimulus token $\left[F_{1}(3,102)=253.1, M S_{\mathrm{e}}=302 ; F_{2}(3,24) 87.5, M S_{\mathrm{e}}=\right.$ $146]$ indicated that the percentage of $b$ responses decreased as the voice onset time of the stimulus increased.

Three effects were significant in the participants analysis only. The main effect of lexical bias was significant $\left[F_{1}(1,34)=16.3, M S_{\mathrm{e}}=1,409\right]$, with more $b$ responses for b- than for p-bias ( $43 \%$ vs. $31 \%$, respectively). Lexical bias interacted with stimulus token $\left[F_{1}(3,102)=8.1\right.$, $\left.M S_{\mathrm{e}}=113\right]$ and showed that the trend for more $b$ responses in the b-lexical-bias words was somewhat larger for positions $2(17 \%)$ and $3(16 \%)$ than for positions $4(10 \%)$ and $5(7 \%)$. The significant effect for lexical carrier $\left[F_{1}(1,34)=7.6, M S_{\mathrm{e}}=185\right]$ showed somewhat more $b$ responses for the flap (39\%) than for the $[\mathrm{t}](36 \%)$ carrier.

A possible concern with interpreting the variant frequency effect is that the p-bias stimulus effect was small (less than half the size of the b-bias effect). Accordingly, a replication with the p-bias (vowel-equated group) stimuli was conducted $(N=24)$. A similar pattern of data was found: The flap carrier had fewer $b$ responses (more identifications that formed a real word), as compared with the [t] carrier (36\% vs. $39 \%$, respectively) and was significant using one-tailed $t$ tests $\left[t_{1}(23)=1.8, S D=\right.$ $\left.8.27, p<.05 ; t_{2}(2)=3.6, S D=1.75, p<.05\right]$.

\section{GENERAL DISCUSSION}

One experiment was reported that investigated whether the frequently heard phonological variant, the American English flap, is lexically represented. The results showed more identification responses forming a real word when the to-be-identified speech sound occurred in the more frequently experienced flap carrier. Phonological variant frequency joins lexical status (Ganong, 1980) and frequency (Connine, Titone, \& Wang, 1993) in influencing phonetic categorization. The phonological variant effect supports the claim that lexical representation of spoken words includes the flap variant. Listeners recognize the flap, in its surface form, via a preexisting representation in memory. Interestingly, some recent experiments have been performed to examine a parallel question with a British English dialect in which the [t] production is the predominant variant (Oxford University undergraduates; D. Patterson, personal communication, November 10, 2003). Patterson compared initial segment identifications $(b$ or $p$ ) for the frequent [t] and infrequent glottal stop carrier words. The results showed more identifications forming a real word for the [t] than for the glottal stop carrier. This finding suggests that the $[\mathrm{t}]$ representation dominates for the British speaker group and is consistent with the present data - the British variant effect is in the direction of the more frequently heard $[t]$.
One potential limitation of the present results is that the frequency structure of flaps has a single dominant production: There are no words from the Patterson and Connine (2001) corpus with frequency statistics showing more [ $\mathrm{t}]$ than flap productions. The frequency distribution of the flap variant precludes a test of a variant frequency effect favoring a [t] carrier. However, research investigating another phonological variant, schwa vowel deletion in poststress position (e.g., the medial vowel of history), addresses this issue. A corpus analysis of schwa vowel deletion frequency (Patterson, LoCasto, \& Connine, 2003) revealed that words vary dramatically in deletion rates and permitted selection of high deletion (greater than 50\%) and low deletion (less than 50\%) rate stimuli. In a set of perceptual experiments (Connine, Ranbom, \& Patterson, 2004), a schwa vowel continuum was used in vowel detection (present/absent) and syllable number judgment (two or three syllables) tasks. An influence of deletion rate was revealed in three aspects of the data. First, low deletion rate words showed more schwa vowel-present judgments (and more three-syllable judgments), as compared with the high deletion rate words. Second, control nonword carriers with the same physical schwa vowel information (and surrounding segments) as their high and low deletion rate word counterparts did not differ. This indicates that the deletion rate effect for words was not a consequence of idiosyncratic properties of the schwa vowel or its environs. Third, both high and low deletion rate words showed more vowelpresent (and three-syllable) judgments, relative to their nonword counterparts, but the difference was larger for the low deletion rate words. Thus, similar to the present results, listeners showed remarkable sensitivity to phonological variant frequency.

The results support the claim that lexical organization capitalizes on systematicity and frequency in representing lexical form. That representation of spoken form utilizes these dimensions is perhaps not surprising, since systematicity and frequency are properties inherent in other aspects of lexical knowledge. Traditional measures of lexical frequency and semantic properties (associative and category structure) of words have long held fundamental roles in the presumed organization of lexical knowledge. The explicit representation of frequently heard phonological variants is also consistent with evidence suggesting that indexical characteristics of voices, as well as within-category information about speech sounds, are encoded by listeners.

The dominance of flapped productions in American English is also consistent with developmental research in which spelling errors have been examined. Treiman, Cassar, and Zukowski (1994) investigated the spelling judgments of young children (beginning readers and spellers) of words with medial flaps (e.g., city) and found that these words were spelled with a medial $d$. Treiman et al.'s findings suggest that early lexical representations for phonological variants are dominated by the more frequent flap production (but see Best, 1994). Furthermore, children's interpretation of a flap in such words as city as 
a voiced stop (spelled as $d$ ) is consistent with what is known about the acoustic-phonetic properties of flaps. Zue and Laferriere (1979) examined the acoustic properties of homophones formed by a flap (e.g., a flapped production of metal vs. medal) and found that [t] and [d] flap durations were identical and generally voiced (both productions had $d$-like properties). Vowel duration measurements in these words showed that $/ \mathrm{t} /$ flaps had shorter (9 msec, on average) preceding vowels (see also Patterson $\&$ Connine, 2001). However, the available evidence indicates that the small differences in vowel duration typically found are not used to distinguish medial flapped /t/ and /d/ (Lorge, 1967; Malécot \& Lloyd, 1968). These data suggest that listeners are unable to distinguish, for example, the flapped production of metal from a production of medal. Furthermore, these results suggest that listeners do not hear consistent acoustic-phonetic markers for interpreting a flapped production of pretty as containing an underlying medial $/ \mathrm{t} /$. These observations are relevant because they raise the question of how the hyperarticulated $[\mathrm{t}]$ variant is represented and processed, an issue as yet not addressed. If listeners recognize the frequently heard flap variant via a prestored representation, how is a hyperarticulated $[\mathrm{t}]$ variant recognized? A strong version of the variant frequency view is that the lexical representation of spoken word variation is guided solely by heard frequency considerations. This would argue that the orthographically consistent $[t]$ variant is not explicitly represented but is recognized via some inference process - a process I have argued against for the recognition of flaps.

An alternative possibility is that lexical representations include both the flap and the [t] variants. If so, how might a rarely heard variant become lexically represented? One possible way that rarely heard word forms might come to be lexically represented is suggested by research on the interdependency of orthographic and phonological lexicons. In the auditory domain, Slowiaczek, Soltano, Wieting, and Bishop (2003) conducted a series of experiments that manipulated graphemic and segmental overlap between prime-target pairs. Lexical decision response times were facilitated when prime-target pairs shared phonological and orthographic information. Slowiaczek et al. argued that orthographic information is activated during spoken word recognition.

In the orthographic domain, interdependencies between auditory and visual domains has long been a focus of reading research. In recent research in which a novel paradigm was used, Inhoff, Connine, and Radach (2002; Inhoff, Connine, Eiter, Radach, \& Heller, 2004) examined the nature of the speech code for potentially flapped words. In the paradigm, participants read sentences in which fixating a target word (e.g., pretty) triggered auditory presentation of the flap, the $[\mathrm{t}]$ form, or a similarsounding word (e.g., gritty). The similar word resulted in increased posttarget reading fixations, but the flapped and the $[\mathrm{t}]$ versions showed no interference. The equivalent effects for flapped and $[\mathrm{t}]$ variants suggests that the speech code in reading reflects both the experienced spoken word environment and orthography-to-phonology mappings. The combined findings across auditory and visual domains indicate strong interdependencies between the reading and the listening lexicons.

The research investigating cross-talk between auditory and written forms raises an interesting possibility for the influence of reading on the processing of spoken language. Specifically, words with highly frequent auditory forms (e.g., a flap) that differ from their orthographic forms may develop parallel representations in the auditory lexicon. For flapped words, this suggests that the $[t]$ version may be represented and activated during spoken word recognition, along with the more frequent flap. Some research has recently shown that a lexically ambiguous flap stimulus (atom pronounced with a flap) may result in activation of an underlying $/ \mathrm{t} /$ representation (McLennan, Luce, \& Charles-Luce, 2003).

In summary, the results provide strong evidence for the claim that representation of auditory form includes explicit representations of the frequently heard variant. Listeners encode surface detail for the speech that they hear and develop lexical representations that match experience. For highly frequent variant forms, explicit representation of phonological variation provides a partial solution for how variants are recognized, without assuming special inference processes. Rather, highly frequent variants are represented and recognized via a match between a lexical representation and the speech input, using the principles of similarity assumed by current models (McClelland \& Elman, 1986; Norris, McQueen, \& Cutler, 2000). One consequence of this view is that theoretical accounts of phonological variant processing will be informed by corpus analyses and variant frequency statistics will serve a critical role in theory development for auditory word recognition.

\section{REFERENCES}

Akmajian, A., Demers, R. A., \& Harnish, R. M. (1984). Linguistics: An introduction to language and communication ( $2 \mathrm{nd}$ ed.). Cambridge, MA: MIT Press.

BEST, C. T. (1994). The emergence of native-language phonological influences in infants. In J. Goodman \& H. Nusbaum (Eds.), The development of speech perception (pp. 167-224). Cambridge, MA: MIT Press.

Connine, C. M., Ranbom, L., \& Patterson, D. (2004). Phonological variant frequency in spoken word recognition: Schwa vowel deletion. Manuscript submitted for publication.

Connine, C. M., Titone, D., \& Wang, J. (1993). Auditory word recognition: Extrinsic and intrinsic effects of word frequency. Journal of Experimental Psychology: Learning, Memory, \& Cognition, 19, 81-94.

GANONG, W. F., III (1980). Phonetic categorization in auditory word perception. Journal of Experimental Psychology: Human Perception \& Performance, $6,110-125$.

Gaskell, M. G., \& Marslen-Wilson, W. D. (1996). Phonological variation and inference in lexical access. Journal of Experimental Psychology: Human Perception \& Performance, 22, 144-158.

GoDFrey, J., MCDANIEL, J., \& Holliman, J. (1992). SWITCHBOARD: A telephone speech corpus for research and development. In ICASSP Proceedings (pp. 517-520). San Francisco.

GoldingER, S. D. (1998). Echoes of echoes? An episodic theory of lexical access. Psychological Review, 105, 251-279. 
Inhoff, A. W., Connine, C. [M.], Eiter, B., Radach, R., \& Heller, D. (2004). Phonological representation of words in working memory during sentence reading. Psychonomic Bulletin \& Review, 11, 320325.

Inhoff, A. W., Connine, C. M., \& Radach, R. (2002). A contingent speech technique in eye movement research on reading. Behavior Research Methods, Instruments \& Computers, 34, 471-480.

LoCasto, P. C., \& Connine, C. M. (2002). Rule-governed missing information in spoken word recognition: Schwa vowel deletion. Perception \& Psychophysics, 64, 208-219.

LoRGE, B. (1967). Production and perception of initial and intervocalic /t/ and /d/ in English. Haskins Laboratory, Status Report on Speech Research, 9, 11-18.

MALÉCOT, A., \& Lloyd, P. (1968). The /t/:/d/ distinction in American alveolar flaps. Lingua, 19, 264-272.

Marslen-Wilson, W., Nix, A., \& Gaskell, G. (1995). Phonological variation in lexical access: Abstractness, inference and English place assimilation. Language \& Cognitive Processes, 10, 285-308.

McClelland, J. L., \& Elman, J. L. (1986). The TRACE model of speech perception. Cognitive Psychology, 18, 1-86.

McLennan, C. T., LuCE, P. A., \& Charles-LuCE, J. (2003). Representation of lexical form. Journal of Experimental Psychology: Learning Memory, \& Cognition, 29, 539-553.

MiLler, J. L. (2001). Mapping from acoustic signal to phonetic cate- gory: Internal category structure, context effects and speeded categorisation. Language \& Cognitive Processes, 16, 683-690.

MullenNiX, J. W. (1997). On the nature of perceptual adjustment to voice. In K. Johnson \& J. W. Mullennix (Eds.), Talker variability in speech processings (pp. 67-83). San Diego: Academic Press.

Norris, D., McQueEn, J. M., \& CutLer, A. (2000). Merging information in speech recognition: Feedback is never necessary. Brain \& Behavioral Sciences, 23, 299-325.

PATTERSON, D., \& CONNINE, C. M. (2001). Variant frequency in flap production: A corpus analysis of variant frequency in American English flap production. Phonetica, 58, 254-275.

Patterson, D., LoCasto, P. C., \& Connine, C. M. (2003). A corpus analysis of schwa vowel deletion frequency in American English. Phonetica, 60, 45-68.

SlowiaczeK, L. M., Soltano, E. G., Wieting, S. J., \& Bishop, K. L. (2003). An investigation of phonology and orthography in spokenword recognition. Quarterly Journal of Experimental Psychology, 56A, 233-262.

Treiman, R., Cassar, M., \& Zukowski, A. (1994). What types of linguistic information do children use in spelling? The case of flaps. Child Development, 65, 1318-1337.

ZuE, V. W., \& LAFERRIERE, M. (1979). Acoustic study of medial /t,d/ in American English. Journal of the Acoustical Society of America, 66, 1039-1050.

\begin{tabular}{|c|c|c|c|c|c|c|}
\hline Voic & & $\begin{array}{r}\text { A } \\
\text { Dur } \\
\text { oker }\end{array}$ & $\begin{array}{l}\text { ND } \\
\text { as } \\
\text { imu }\end{array}$ & neti & Sti & \\
\hline & & & Stim & oker & & \\
\hline Item & 1 & 2 & 3 & 4 & 5 & 6 \\
\hline Party & 4 & 30 & 35 & 40 & 45 & 92 \\
\hline Pattern & 12 & 27 & 33 & 38 & 43 & 91 \\
\hline Pretty & 13 & 36 & 41 & 46 & 51 & 81 \\
\hline Beetle & 1 & 18 & 22 & 27 & 32 & 86 \\
\hline Better & 5 & 26 & 31 & 37 & 42 & 63 \\
\hline Bottle & 8 & 26 & 32 & 37 & 42 & 73 \\
\hline
\end{tabular}

(Manuscript received July 18, 2003; revision accepted for publication February 26, 2004.) 